\title{
ARTICLE \\ Cerebral white matter sex dimorphism in alcoholism: a diffusion tensor imaging study
}

\author{
Kayle S. Sawyer (iD) ${ }^{1,2,3,4}$, Nasim Maleki ${ }^{5}$, George Papadimitriou ${ }^{6}$, Nikos Makris ${ }^{6}$, Marlene Oscar-Berman ${ }^{2,7}$ and Gordon J. Harris $^{8}$
}

Excessive alcohol consumption is associated with brain aberrations, including abnormalities in frontal and limbic brain regions. In a prior diffusion tensor magnetic resonance imaging (dMRI) study of neuronal circuitry connecting the frontal lobes and limbic system structures, we demonstrated decreases in white matter fractional anisotropy in abstinent alcoholic men. In the present study, we examined sex differences in alcoholism-related abnormalities of white matter connectivity and their association with alcoholism history. The dMRI scans were acquired from 49 abstinent alcoholic individuals ( 26 women) and 41 nonalcoholic controls ( 22 women). Tract-based spatial statistical tools were used to estimate regional FA of white matter tracts and to determine sex differences and their relation to measures of alcoholism history. Sex-related differences in white matter connectivity were observed in association with alcoholism: Compared to nonalcoholic men, alcoholic men had diminished FA in portions of the corpus callosum, the superior longitudinal fasciculi II and III, and the arcuate fasciculus and extreme capsule. In contrast, alcoholic women had higher FA in these regions. Sex differences also were observed for correlations between corpus callosum FA and length of sobriety. Our results suggest that sexual dimorphism in white matter microstructure in abstinent alcoholics may implicate underlying differences in the neurobehavioral liabilities for developing alcohol abuse disorders, or for sequelae following abuse.

Neuropsychopharmacology (2018) 43:1876-1883; https://doi.org/10.1038/s41386-018-0089-6

\section{INTRODUCTION}

Excessive alcohol consumption is associated with abnormalities in brain regions involved in cognition and emotion, as evidenced by multiple neuroimaging, post-mortem, and neurobehavioral studies, even in the absence of substantial global intelligence deficits $[1,2]$. However, the majority of neuroscientific studies in alcoholism have focused on men [3,4], and there is limited scientific literature evaluating sex-specific similarities and differences in brain pathology associated with alcoholism. Importantly, research comparing men and women is valuable for validating published findings, interpreting the results, and drawing scientific conclusions. Moreover, there is accumulating evidence of sex differences in pharmacokinetic responses to alcohol consumption and metabolism $[5,6]$, which may further impact sex differences in health problems mediated by social and environmental, as well as neurobiological, physiological, and genetic influences [7]. In other words, the findings in men may not accurately translate or be extrapolated to women, and there may be meaningful sex differences. These sex differences are of particular interest to translational researchers who investigate how factors such as sex, age, and comorbidities can be used clinically in diagnosing asymptomatic at-risk individuals, and to inform individual treatment strategies targeting recognized neural vulnerabilities [8].

Multiple studies, focused mainly on ALC men, have reported significant structural brain abnormalities that are correlated with indices of alcoholism history, such as the duration of alcoholism, the amount of alcohol consumed, or the duration of abstinence $[9,10]$. These abnormalities involve cortical gray matter, as well as white matter fiber tracts, and may appear before any behavioral symptoms arise. Alcoholism is particularly damaging to cerebral white matter, as has been revealed by post-mortem neuropathology [11-13] and by in vivo structural MRI studies [14, 15]. Consistent with these findings, post-mortem RNA analyses of superior frontal lobe samples found that genes related to myelin structure were down-regulated in alcoholics [16]. Interestingly, sex-specific differences in the expression of genes that are significantly influenced by chronic alcohol abuse has been reported in the postmortem prefrontal cortex of subjects with alcohol dependence [17] although their exact relation to myelination is not completely clear [18].

In our prior work, we used diffusion tensor magnetic resonance imaging (dMRI) to examine the integrity of white matter fiber tracts in the brains of abstinent ALC men compared with nonalcoholic control (NC) men [19]. We found that the ALC men had lower fractional anisotropy (FA) than NC men within several white matter fiber tracts. In another study we found that ALC men had smaller volumes of brain regions in the extended reward and oversight system [20]. Later, however, we identified sex dimorphism both in the regional white matter volumes in ALC men and women [21], and also in the size of reward system regional volumes [22]. In the present study, we examined sex-specific

\footnotetext{
${ }^{1}$ Department of Anatomy and Neurobiology, Boston University School of Medicine, Boston, MA 02118, USA; ${ }^{2}$ VA Boston Healthcare System, Boston, MA 02130, USA; ${ }^{3}$ Athinoula A. Martinos Center, Massachusetts General Hospital, Boston, MA 02114, USA; ${ }^{4}$ Sawyer Scientific, LLC, Boston, MA 02120, USA; ${ }^{5}$ Department of Radiology, Massachusetts General Hospital, Boston, MA 02114, USA; ${ }^{6}$ Departments of Neurology, Psychiatry, and Radiology, Center for Morphometric Analysis, and Athinoula A. Martinos Center, Massachusetts General Hospital, Boston, MA 02114, USA; ${ }^{7}$ Departments of Neurology, Psychiatry, Anatomy and Neurobiology, Boston University School of Medicine, Boston, MA 02118, USA and ${ }^{8}$ Radiology Computer Aided Diagnostics Laboratory, Center for Morphometric Analysis, Massachusetts General Hospital, Boston, MA 02114, USA Correspondence: Kayle S. Sawyer (kslays@bu.edu)
}

Received: 8 December 2017 Revised: 23 March 2018 Accepted: 5 May 2018

Published online: 09 May 2018 
differences in the impact of alcoholism. In doing so, we sought first to confirm our prior findings of lower FA in white matter fiber tracts in abstinent ALC men in an independent sample, and subsequently, to compare these white matter abnormalities to those of abstinent ALC women. Based on our prior reports of sex dimorphism in alcoholic brains, we hypothesized that sex-related differences in white matter fiber tracts would vary between the ALC and NC groups, and the present study represents the first voxel-wise whole-brain investigation of how sex impacts FA differences between those groups.

\section{MATERIALS AND METHODS}

\section{Participants}

Participants were solicited through online advertisements (e.g., Craigslist), newspaper advertisements (e.g., Boston Metro), and flyers posted at participating institutions (e.g., Boston Medical Center and VA Hospital bulletin boards), and in public places (e.g., churches, telephone poles, stores). The study included 49 ALC participants ( 26 women), and 41 NC participants ( 22 women). They were right-handed, fluent English speaking individuals who ranged in age from 23 to 76 years. The groups were age balanced and had comparable socioeconomic backgrounds, and ethnic distributions (ALC: 1 American Indian, 7 Black, 41 White; NC: 1 Asian, 11 Black, 1 Hispanic, 28 White). The ALC participants met DSM-IV criteria for alcohol abuse or dependence, and had a minimum duration of five years of heavy drinking (21 or more drinks per week). As detailed in the Results section, all four subgroups performed comparably on neurobehavioral screening tests; the source data and code are provided in the Supplemental Dataset S1 and Supplemental Code S1. The research was approved by the Institutional Review Boards of Boston University School of Medicine, VA Boston Healthcare System, and Massachusetts General Hospital. Participants were reimbursed for their time and travel expenses.

\section{Inclusion and exclusion criteria}

Selection procedures included structured interviews to determine education level, health history, and history of alcohol and drug use. Individuals were excluded from further participation if any source indicated one of the following: Korsakoff's syndrome; HIV; cirrhosis; major head injury with loss of consciousness $>30$ minutes; stroke; epilepsy or seizures unrelated to alcoholism; Hamilton Rating Scale for Depression (HRSD; [23]) score over 18; schizophrenia; or electroconvulsive therapy. Additionally, we excluded individuals who failed screening for MRI safety (e.g., metal implants, obesity, pregnancy). Further exclusion of participants took place during the preprocessing of the MRI data for analysis, if they did not complete the MRI scan; showed excessive head motion and instability during the scan; or had a brain abnormality discovered by the scan.

The ALC individuals were included if they had a history of five or more years of drinking 21 or more drinks per week, a level considered as heavy drinking by Cahalan and colleagues (1969), and an approach that is consistent with later quantitative assessments [24]. These sample characteristics are concordant with our previous studies and allow direct comparisons with prior work from this laboratory. ALC individuals also met Diagnostic and Statistical Manual (DSM-IV) [25] criteria for alcohol abuse or dependence, and had been abstinent for at least four weeks before testing and scanning, with the exception of one man who drank the prior weekend, one man who drank three weeks before the scan, and one woman who drank six days before the scan. These three participants were included because all of them reported that the drinking occurred only once, and they remained abstinent thereafter. A comprehensive psychiatric interview (described below) indicated that none of the participants had current drug abuse or dependence for any illicit substance including marijuana. Moreover, none of the participants had used illicit drugs for the four years preceding enrollment, with the exception of one ALC woman who stopped occasional marijuana use six months prior to testing, and one ALC man who stopped occasional marijuana use two months prior to testing. These two participants were included because both of them had stopped their occasional use which we determined was infrequent.

\section{Evaluation of drinking history}

As described above, drinking history was evaluated using a standardized alcohol use questionnaire [26] that yielded three measures: (1) length of sobriety (LOS); (2) duration of heavy drinking (DHD), i.e., the number of years consuming $\geq 21$ drinks per week (one drink: $355 \mathrm{~mL}$ beer, $148 \mathrm{~mL}$ wine, or $44 \mathrm{~mL}$ hard liquor); and (3) a quantity frequency index, roughly corresponding to number of daily drinks (DD; one ounce of ethanol per drink). The DD measure evaluated the amount, type, and frequency of alcohol usage over the last six months (for NC participants), or over the six months preceding cessation of drinking (for ALC participants). To ensure that DD was comparable between ALC participants with complete abstinence, and four ALC who had quit but then drank occasionally ( $\mathrm{DD}<3.0$ ), we estimated DD based on the last six months of heavy drinking in the latter group. Thus, the measure consistently reflected DD across all ALC participants.

Neurobehavioral and psychiatric evaluations

In order to minimize confounding effects from illicit drug use, psychoactive prescription drug use, and psychiatric comorbidity, participants were given a battery of screening tests, one of which was a computer-assisted, shortened version of the Diagnostic Interview Schedule [27] that provided lifetime psychiatric diagnoses according to DSM-IV criteria [25]. Additionally, tests of intelligence, memory, and affect were administered, including the Wechsler Adult Intelligence Scale, Third Edition (WAIS-III) for FullScale IQ (FSIQ), Verbal IQ (VIQ), and Performance IQ (PIQ); the Wechsler Memory Scale, Third Edition (WMS-III) for Immediate Memory, Delayed Memory, and Working Memory [28]; and the HRSD to assess depression. Neurobehavioral and psychiatric evaluations were extensive and typically required six to nine hours over three or more days.

For neurobehavioral, psychiatric, and drinking variables, differences in diagnostic Group (ALC vs. NC) and Sex (men vs. women) were assessed using two-tailed Welch two sample $t$-tests on each of the measured indices in each category.

\section{Diffusion imaging}

Diffusion-weighted dMRI images were acquired on a 3 Tesla Siemens Tim Trio scanner to compute quantitative white matter diffusivity measures. A 12 minute 60 -direction dMRI scan was obtained with $2 \times 2 \times 2 \mathrm{~mm}$ resolution at $b=700 \mathrm{~s} / \mathrm{mm}^{2}$ (number of T2 b0 images $=10, T E=94 \mathrm{~ms}, \mathrm{TR}=9800 \mathrm{~ms}$, interleaved axial slices $=64, \quad F O V=256 \mathrm{~mm}, \quad$ matrix $=128 \times 128$, skip $=0 \mathrm{~mm}$, bandwidth $=1860 \mathrm{~Hz} /$ pixel). For individual single-subject level and group-level comparisons of the dMRI processing stream, FSL v5.0 [29] Tract-Based Spatial Statistics (TBSS v1.1, www.fmrib.ox.ac. uk/fsl/TBSS) were used. We applied Threshold Free Cluster Enhancement (TFCE), a stringent control for Type I family-wise error rate. This method corrects for multiple comparisons and eliminates the need for setting an arbitrary cluster-forming threshold by merging voxel-wise statistics with local spatial neighborhood information [30]. Details on the diffusion imaging processing stream are provided in Supplementary Methods.

We used a general linear regression model with three contrasts to examine: Group effects (ALC vs. NC) for men, Group effects for women, and interactions between Group and Sex. Next, individual subjects' average FA values were extracted from each cluster with significant Group by Sex interactions, and subsequent ad hoc analyses were performed, as described in the Supplementary 
Table 1. Participant demographic characteristics and drinking histories

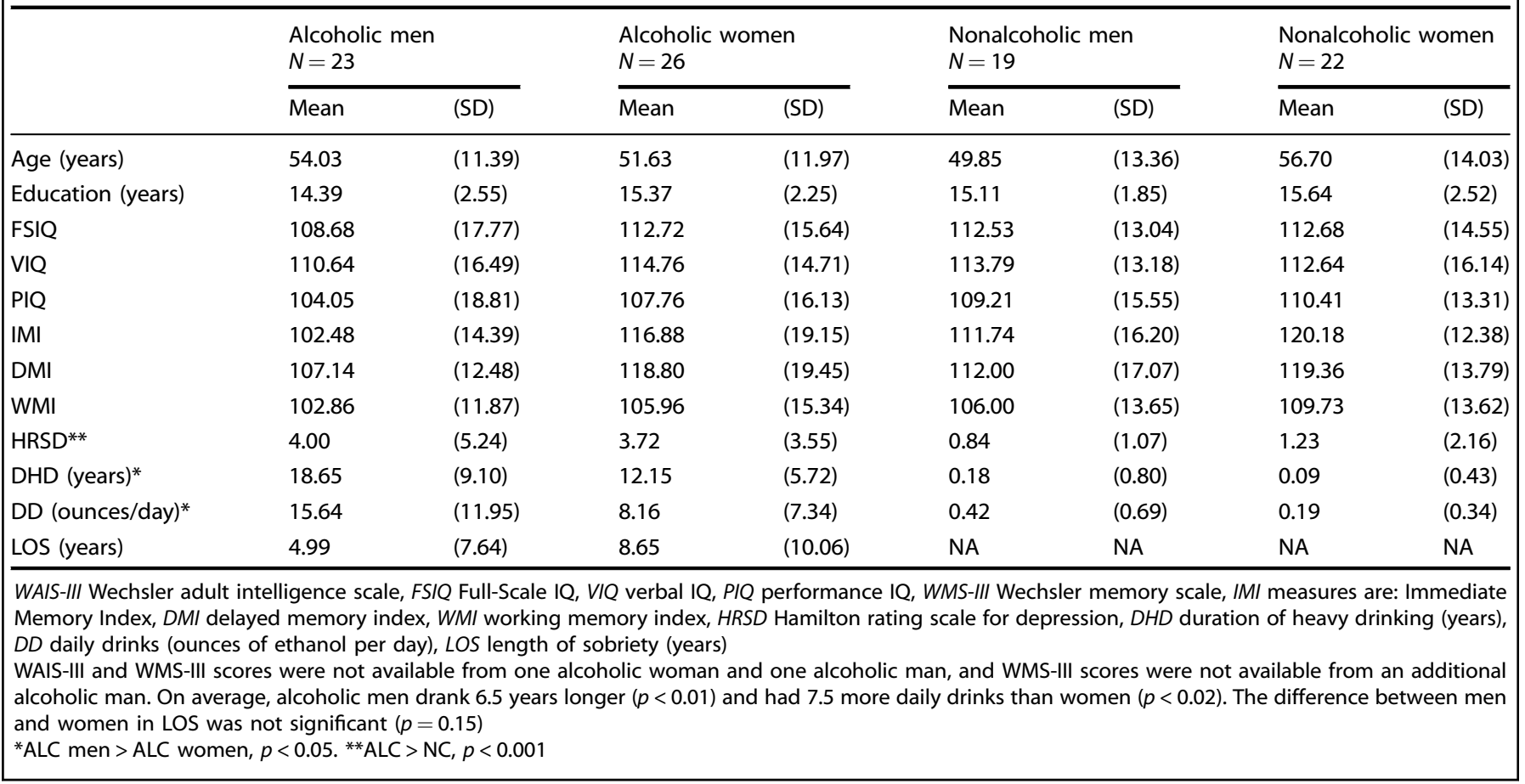

Methods. In addition, we examined the relationships of FA from these clusters to drinking history and memory scores.

\section{RESULTS}

Neurobehavioral, psychiatric, and drinking measures

There were no significant differences between the ALC and NC participants with respect to age, education, WAIS-III, and WMS-III indices. There also were no significant Group by Sex interactions or main effects with respect to age, IQ, memory scores, and education (Table 1; ranges are presented in Table S1). Although the depression scores for the ALC group were significantly higher than for the NC group $(p<0.001)$, both groups' scores were low (mean 3.9 vs. 1.0): HRSD scores of 8,16 , and 25 or above indicate mild, moderate, or severe depression, respectively [31]. The source data and code for these data are provided in the Supplemental Dataset S1 and Supplemental Code S1.

In order to account for any potential biases or confounding induced by sex differences in drinking histories, we performed a subanalysis $(N=81)$ for which ALC men and women had comparable drinking history (i.e., there were no significant differences between them). The TFCE corrected FA cluster volumes obtained from the interaction effect for this subgroup analysis overlapped with $98 \%$ of the cluster volumes obtained from the entire group, indicating the subgroup results were consistent with the entire group (as presented below).

\section{Fractional anisotropy}

There were no significant results from the axial or radial diffusivity analyses. For FA however, three significant white matter tract clusters displayed Group by Sex interactions ( $p<0.05$ using TFCE). The clusters were overlaid with a white matter atlas [32] and examined by a skilled neuroanatomist (N.M.). The clusters are shown in Fig. 1 and their anatomical locations are described in Table 2 (non-thresholded contrasts are shown in Fig. S1). The clusters included the genu and anterior parts of the body of the corpus callosum (CC2, CC3, and CC4), the left arcuate fasciculus and extreme capsule (AF and $\mathrm{EmC}$ ) [33], and the left superior longitudinal fasciculi (SLFII and SLFIII) [34]. Of note regarding the corpus callosum, TFCE correction at first revealed two clusters (CC2 and CC3; $517 \mathrm{~mm}^{3}$ ) and $\left(C C 4 ; 4 \mathrm{~mm}^{3}\right.$ ) separated by $6.1 \mathrm{~mm}$. Our neuroanatomist (N.M.) determined that CC2, CC3, and CC4 were within the corpus callosum, and if we were to have used an adjusted threshold of $p<0.06$, the two clusters would have joined to form a single cluster. Therefore, the two clusters were combined into one $521 \mathrm{~mm}^{3}$ cluster.

The relationships between groups with mean FA in these clusters are shown in Fig. 2 and Table S2 (Table S3 shows the FA ranges). Similar interactions were observed for all three clusters: ALC men had lower FA than NC men, while ALC women had higher FA than NC women (all Welch's $t$-test $p<0.05$ ).

In addition to assessing Group by Sex interaction effects, we examined group differences for men and women separately in our TBSS model (Table 2). For women, no clusters were significant after TFCE correction. For men, one cluster was identified, as shown in Fig. S2 and Fig. S3. This cluster was superior to the posterior horn of the left lateral ventricle, and included the posterior thalamic radiation (PTR). For the PTR cluster, ALC men had 0.07 lower FA than NC men (Table S2).

Behavioral correlations with regional FA measures

We examined the relationship of the three drinking history measures (DHD, DD, and LOS) to FA for the three interaction clusters we identified (Tables S4A-S4C). Further, we examined the interaction of Sex with each drinking history measure to determine how these relationships differed for alcoholic men and women. A significant interaction with Sex was found for the CC2, CC3, and CC4 cluster: FA was positively associated with LOS for men, while no significant relationship was found for women (Fig. 3 and Table S4C).

In addition, for the total sample of 90 participants, we examined the relationships of the mean FA of each cluster (Tables S5) to (1) age at scan, and (2) memory scores (IMI, DMI, and WMI). FA declined with age by 0.001 per year in the SLF II and SLF III clusters $(p<0.05)$, and 0.002 per year, both in the AF and EmC cluster, and in the CC2, CC3, and CC4 cluster $(p<0.001)$. IMI was associated 


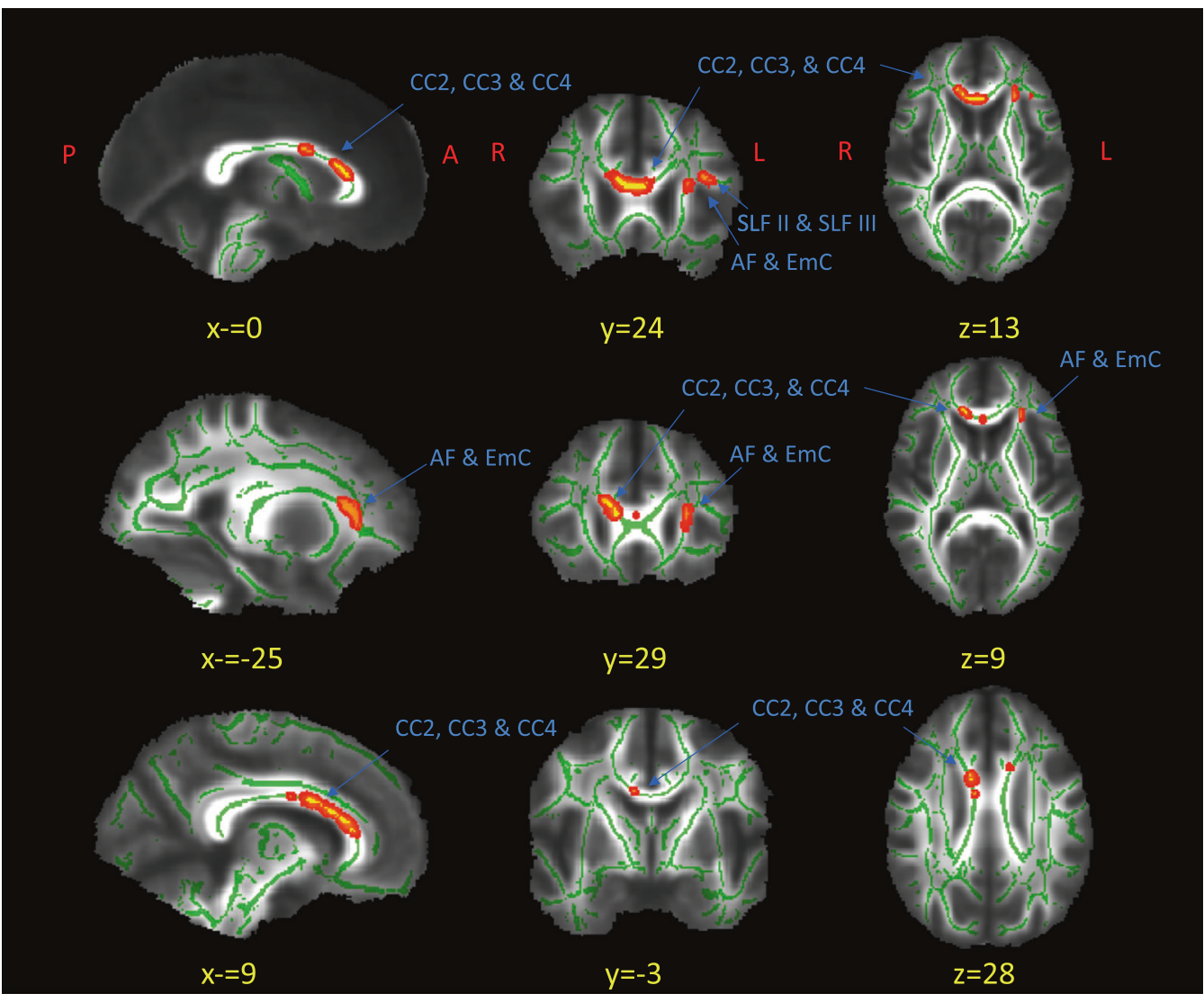

Fig. 1 Significant Group by Sex interaction in FA differences in white matter. The maps represent significant ( $p<0.05$, TFCE corrected) clusters from voxel-based FA Group by Sex interaction (red-yellow) in sagittal, coronal, and axial views. The green represents the skeletonized mean FA map of all of the subjects, which has been overlaid on a mean FA map of all the subjects. The red-yellow clusters represent regions with significant Group by Sex interactions. Three clusters were located as follows: the anterior portions and body of the corpus callosum (CC2, CC3, and CC4); the left superior longitudinal fasciculi (SLF II and SLF III); and the left arcuate fasciculus and extreme capsule (AF and EmC); see Fig. 2 and Table S2 for FA levels

with higher FA for the CC2, CC3, and CC4 cluster $(p<0.05)$, while $\mathrm{DMI}$ and WMI were not significantly related to FA in any cluster. A significant interaction of Group and Sex was observed with Age for the CC2, CC3, and CC4 cluster $(p<0.05)$, where ALC men $(p<0.05)$, NC men $(p<0.01)$, and NC women $(p<0.001)$ had negative relationships with Age, while ALC women did not (Table S5A). No significant Group or Sex interactions with IMI, DMI, or WMI were observed for the relationship of FA for any of the three clusters.

\section{DISCUSSION}

While the effects of long-term chronic alcoholism on the brain have been examined in neuroimaging studies, most have included only or mainly men, or did not differentiate between men and women in a mixed sample. These studies generally have reported brain atrophy, cortical thinning, and smaller volumes of the frontal lobes, hippocampus, thalamus, pons, and cerebellum, with increases in the ventricles and cerebrospinal fluid in men. DTI abnormalities reported in those prior studies have mainly encompassed fronto-cerebellar circuits, genu and splenium of the corpus callosum, the centrum semiovale, the superior longitudinal fasciculus, the external capsule, the fornix, and the cingulum [19, 35-37].

We had previously examined differences in cerebral white matter integrity between abstinent ALC and NC men [19]. In the present study, we aimed to examine sex-specific differences in the impact of alcoholism, and potentially underlying sex dimorphic brain differences in the predisposition to alcoholism. The results showed sex-related abnormalities in the white matter tracts of the alcoholic individuals. The abstinent alcoholic men had white matter FA deficits consistent with our prior study, but the abstinent ALC women did not. These distinct patterns of white matter anomalies may suggest a different underlying neural basis for sex-specific propensity (i.e., differential risk factors) and/or sequelae to long-term alcoholism, and have implications for further translational and clinical investigations of possible sexspecific approaches to prevention and treatment [63, 65, 66, 4].

The exact nature of pathophysiological white matter changes underlying the observed differences in the current study cannot be inferred from DTI results alone. However, recent findings using nonhuman animal models of alcoholism may help to explain alcohol induced white matter deficits. Animal studies have provided morphological evidence that chronic alcohol exposure can cause damage not only to nerve cells but also to nerve fibers [38-40].

In the present study, sex differences in the relationship of FA with alcoholism were observed for three clusters: the anterior and middle portions of the corpus callosum; the arcuate fasciculus and extreme capsule; and the superior longitudinal fasciculi II and III (Fig. 1). As shown in Fig. 2, ALC men had lower FA than NC men, while ALC women had higher FA than NC women. Parallel gender dimorphism was reported in left frontal cortical thickness in adolescent binge drinkers [41], which may suggest that these gender differences may pre-date long-term alcoholism effects, and may reflect differential pre-existing factors, such as personality, prodromal psychiatric comorbidity, or motivational factors for drinking. In other words, it may be that drinking problems arise 
Table 2. Significant clusters from FA comparisons

\begin{tabular}{lllllll}
\hline Contrast & Cluster \# & Cluster size $\left(\mathbf{m m}^{\mathbf{3}}\right)$ & TalX & TalY & TalZ & Annotation \\
\hline Group by sex interaction & 1 & 40 & -33 & 22 & 17 & SLF II and SLF III \\
Group by sex interaction & 2 & 69 & -24 & 26 & 10 & AF and EmC \\
Group by sex interaction & 3 & 521 & 0 & 22 & 13 & CC2, CC3, and CC4 \\
FALC vs. FNC & $\mathrm{ns}$ & $\mathrm{ns}$ & $\mathrm{ns}$ & $\mathrm{ns}$ & $\mathrm{ns}$ & $\mathrm{ns}$ \\
MALC vs. MNC & 4 & 271 & -29 & -56 & 15 & PTR \\
\hline
\end{tabular}

AF arcuate fasciculus, SLFII and SLFIII left superior longitudinal fasciculi, EmC extreme capsule, CC2 and CC3 anterior portions of the corpus callosum, CC4 body of the corpus callosum, PTR posterior thalamic radiation

After threshold-free cluster enhancement multiple-comparison correction comparing Group by Sex interactions for FA differences, three clusters were identified as significant (Fig. 1 for anatomical locations, Fig. 2 for FA levels, and Table S2 for mean cluster values). For the MALC vs. MNC comparison, one significant cluster was identified; no significant clusters were found for FALC vs. FNC (marked "ns"). TalX, TalY, and TalZ represent the Talairach coordinates of the most significant voxel in the cluster. The coordinates were translated to the Talairach coordinates using Yale's Biolmage Suite [59-67]

in men and women for different reasons [4, 41, 42], and those associated risk factors could be manifested by differential prodromal FA abnormalities such as the motivation to drink, or personality factors [43]. In any case, it is important to note, that variables such as trait characteristics or behavioral disorders influencing the development of substance use disorders and/or increasing the sensitivity to alcohol's effects on the brain, do not mediate risk only in one sex. These relationships are testable and may serve to differentiate sex differences from other psychosocial risk factors.

For the first cluster, the anterior corpus callosum conveys interhemispheric signals across prefrontal brain regions [32]. In a previous study [22], the volume of dorsolateral prefrontal cortex was reported to be smaller in ALC men than NC men, while the opposite was observed for women, perhaps reflecting sex differences in alcoholism-induced effects on the cortex that are concordant with our white matter findings in the current study. Further, the volume of the mid-anterior corpus callosum was smaller in ALC men than NC men [21]. Considering the inherently specialized functions of the two human frontal lobes, the axonal abnormalities we observed in the corpus callosum could be tied to alcoholism-related abnormalities observed in personality [44] or psychiatric conditions [45].

For the second cluster, a significant alcoholism Group by Sex interaction was observed in the anterior portion of the arcuate fasciculus and extreme capsule. While the arcuate fasciculus is thought to conduct signals primarily between Broca's area and Wernicke's area, this interpretation remains unsettled [46], and recent research suggests broader connectivity [47]. While one might predict that the reduced connectivity observed in ALC men could impart a reduction in language ability, we did not observe one, nor have language deficits been widely reported [2]. The cluster also might include the extreme capsule, which may be involved in long-term semantic memory. However, caution is advised when interpreting dMRI results for this tract [47].

For the third cluster, we observed a similar pattern of FA abnormalities for a region including portions of two major longitudinal pathways, SLF II and SLF III, which have been implicated in diverse functions including working memory [47]. Working memory deficits in alcoholism are common [2]. Clearly, further research, including the use of fMRI paradigms [48, 49], could illuminate the relationship of these abnormalities to sex, prodromal brain abnormalities, and preexisting risk factors. As such, new findings can present a striking clinical target for the treatment or prevention of alcoholism.

By examining the amount and duration of drinking, along with the duration of abstinence, in relation to the present dMRI findings, we can begin to suggest some consequences of drinking. We found that LOS was correlated with higher FA of the callosal white matter in men, but not for women (Fig. 3). This interaction could indicate more recovery for men, considering our observation that the men with short sobriety periods had lower FA than women with short sobriety periods, but the opposite was true for long sobriety periods. In a recent study [50] on a large sample of non-abstinent, heavily drinking alcoholics (324 participants, 30\% female), more frequent drinking (a close match to our estimates of DD during the period of heavy drinking) was reported to contribute to lower FA in ALC women but not ALC men-even though both sexes were similar in terms of demographics, history of drinking, and drinking severity. Since our alcoholic participants were abstinent for years before participating in our study (see Table 1), the direct comparison between the findings of our study and this study is not possible. However, this study also similarly reveals sex-related differences in alcohol-related effects on white matter integrity. A previous study reported lower FA values in ALC women as compared to ALC men, when both groups were matched by the amount of lifetime alcohol consumption and length of abstinence [15]. The ALC men and women in our study were not matched for either of these variables; however, our findings were consistent when we analyzed a subgroup that included men and women with comparable drinking histories.

As aging also has been associated with a decline in the integrity of white matter microstructure [51, 52], we examined the relationship of age to the three clusters with significant Group by Sex interactions. Age was correlated with lower FA in each cluster, at levels comparable to those previously reported $(-0.002$ to -0.001 per year).

\section{Limitations}

Although we found strong evidence for alcoholism sex differences in white matter integrity, this study had several limitations. First, as noted above, our sample of ALC women had shorter (by 6.5 years) and less severe (7.5 fewer DD) drinking histories than the ALC men. However, when we analyzed subgroups with comparable drinking histories, we found that the clusters remained significant. Second, although the Diagnostic Interview Schedule [27] assessed nicotine dependence in our sample (16 positive, 70 negative, 4 missing), only five individuals were currently nicotine dependent, and we did not have quantitative measures of nicotine usage for all participants (e.g., age of onset, chronicity, cigarettes/day, and abstinence; see Supplemental Dataset S1). Cigarette smoking is common among alcoholics, may interact with aging effects, and is known to impact brain structure and function as a main and interactive effect $[53,54]$. Nor did we counterbalance recruitment by the participants' family history of alcoholism (see Table S6), so the differences in FA we observed may be related to heritable traits. Moreover, there is evidence that in addition to family history, binge drinking also contributes to the impact of alcoholism [55]. Although we required consistency in the pattern of drinking (at least 21 drinks/week on average for at least five 
0.9

$\mathrm{CC} 2, \mathrm{CC} 3, \& \mathrm{CC} 4$

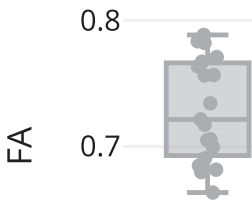

0.6

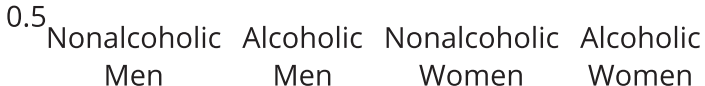

SLFII \& SLFIII

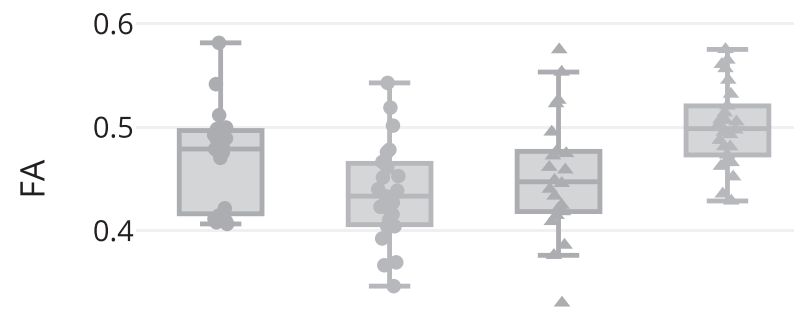

0.3

$\begin{array}{ccc}\begin{array}{c}\text { Nonalcoholic } \\ \text { Men }\end{array} & \text { Men } & \text { Nonalcoholic Alcoholic } \\ \text { Women } & \text { Women }\end{array}$

0.6

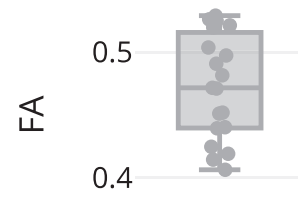

0.3
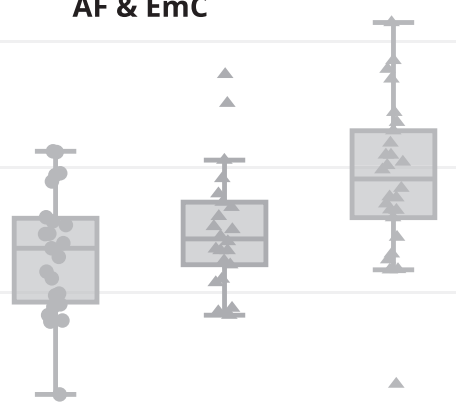

$\Delta$

Fig. 2 The relationship of alcoholism and fractional anisotropy (FA) differs for men and women. In a significant two-way interaction of Group by Sex, three clusters were identified after threshold-free cluster enhancement correction for multiple comparisons $(p<0.05)$. ALC men had lower FA than NC men, while ALC women had higher FA than NC women $\left({ }^{*} p<0.05 ;{ }^{* *} p<0.01\right)$. The three clusters were located as follows: the anterior portions and body of the corpus callosum (CC2, CC3, and CC4); the left superior longitudinal fasciculi (SLF II and SLF III); and the left arcuate fasciculus plus extreme capsule (AF and EmC); see Fig. 1 for cluster locations and Table S2 for mean values

years), it is possible that some participants had a drinking pattern that might be considered binge drinking.

Another element of alcoholism that may differentiate individuals is whether or not they sought formal treatment to initiate their recovery [56]. In our study, $90 \%$ of our ALC sample (24

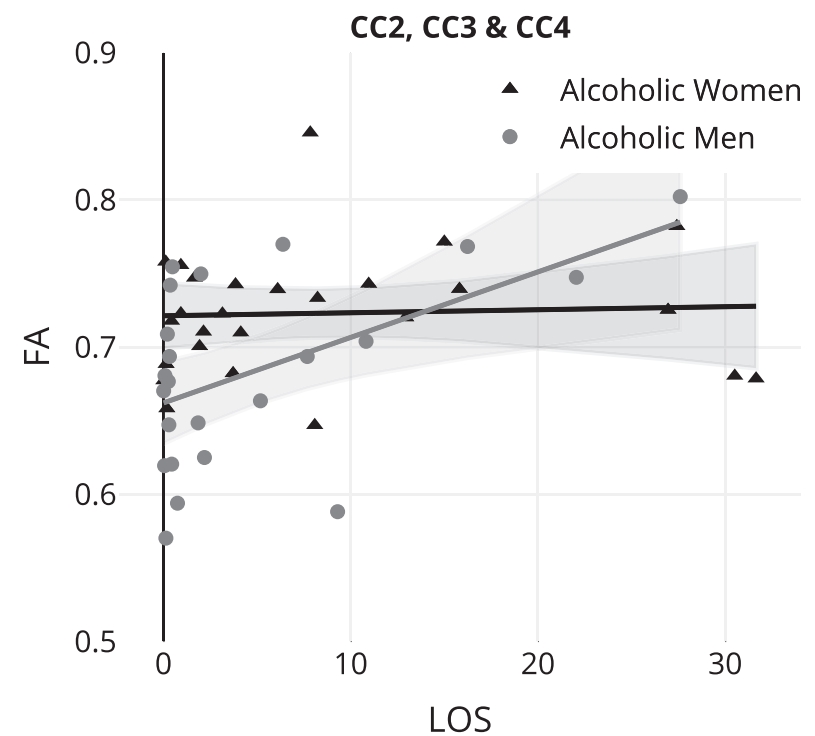

Fig. 3 Fractional anisotropy is related to drinking history differently in men and women with alcoholism. The CC2, CC3, and CC4 cluster showed a significant interaction effect in the relationship between Length of Sobriety (LOS) and Sex; see Table S4C. Regression lines and $95 \%$ confidence intervals are displayed separately for men and women. Women are displayed with triangles and men are displayed with circles

women, 20 men) sought treatment-including Alcoholics Anonymous-for their drinking. Such persons may differ from those who initiate recovery without seeking formal treatment. Third, the ALC and NC groups had relatively high Full Scale IQ scores. This has been observed in other of our studies that recruit participants in the greater Boston area [21, 57, 58], but it may limit the generalizability of our findings. Finally, the cross-sectional nature of this study hinders causal interpretation; that is, we cannot determine whether the FA differences predated the onset of drinking, or were caused by heavy drinking and associated sequelae. Nonetheless, the findings presented here confirm and extend the results found in ALC men to a sample of ALC women, and broaden the knowledge base of sex differences in relation to the psychobiology of alcohol use disorders.

\section{CONCLUSIONS}

This DTI study provides evidence in support of a growing understanding of the nature of sex dimorphism in brain and behavioral abnormalities associated with alcoholism. Specifically, we found differences between alcoholic men and women in white matter integrity, evidenced by lower FA values in ALC men and higher FA in ALC women. The white matter abnormalities in men observed in our study support previous literature on alcoholism, specifically those studies indicating damage to cortical and limbic brain regions that comprise a system essential for normal emotional functioning, and modulating the effectiveness of positive and negative reinforcement in human behavior. Future research is needed to explore whether sexual dimorphism in white matter microstructure may implicate underlying differences in the neurobehavioral liabilities for developing alcohol abuse disorders, or for sequelae following abuse.

\section{ACKNOWLEDGEMENTS}

We thank Elinor Artsy, Sheeva Azma, Julie Howard, Maaria Kemppainen, Diane Merritt, Alan Poey, Daniel Salz, Yulia Spantchak, Trinity Urban, Susan Mosher Ruiz, Maria Valmas, and Robert Zondervan for assistance with activities such as recruitment, assessment, and neuroimaging of the research participants. We also 
thank the reviewers for their helpful suggestions. Funding and Disclosure: This work was supported in part by grants from: National Institute on Alcohol Abuse and Alcoholism (NIAAA) R01AA07112 and K05AA00219, and by the US Department of Veterans Affairs Clinical Science Research and Development grant I01CX000326 to Dr. M.O.B.; the National Institute on Aging (NIA) and National Institute of Mental Health (NIMH) grant R01AG042512 and the National Center for Complementary and Integrative Health (NCCIH) grant R21AT008865 to Dr. N.M.; the Center for Functional Neuroimaging Technologies grant P41RR14075; and instrumentation grants 1S10RR023401, 1S10RR019307, and 1S10RR023043 from the National Center for Research Resources (now National Center for Advancing Translational Sciences). The content is solely the responsibility of the authors and does not necessarily represent the official views of the National Institutes of Health, the U.S. Department of Veterans Affairs, or the United States Government.

\section{ADDITIONAL INFORMATION}

Supplementary information accompanies this paper at (https://doi.org/10.1038/ s41386-018-0089-6)

Competing interests: The authors declare no competing interests.

Publisher's note: Springer Nature remains neutral with regard to jurisdictional claims in published maps and institutional affiliations.

\section{REFERENCES}

1. Oscar-Berman M, Marinković K. Alcohol: effects on neurobehavioral functions and the brain. Neuropsychol Rev. 2007;17:239-57.

2. Oscar-Berman M, Valmas MM, Sawyer KS, Ruiz SM, Luhar RB, Gravitz ZR. Profiles of impaired, spared, and recovered neuropsychologic processes in alcoholism. Handb Clin Neurol. 2014;125:183-210.

3. Ruiz SM, Oscar-Berman M. Closing the gender gap: the case for gender-specific alcoholism research. J Alcohol Drug Depend. 2013;1:1-3.

4. Ruiz SM, Oscar-Berman M. Gender and alcohol abuse. In: Martin SC, editor. The SAGE Encyclopedia of Alcohol: Social, Cultural, and Historical Perspectives. Vol 2. Los Angeles: Sage Publications; 2015. p. 586-91

5. Chrostek L, Jelski W, Szmitkowski M, Puchalski Z. Gender-related differences in hepatic activity of alcohol dehydrogenase isoenzymes and aldehyde dehydrogenase in humans. J Clin Lab Anal. 2003;17:93-6.

6. Ramchandani VA, Bosron WF, Li TK. Research advances in ethanol metabolism. Pathol Biol. 2001;49:676-82.

7. Verhulst B, Neale MC, Kendler KS. The heritability of alcohol use disorders: a metaanalysis of twin and adoption studies. Psychol Med. 2015;45:1061-72.

8. Greenfield SF, Grella CE. Alcohol \& drug abuse: What is "womenfocused" treatment for substance use disorders? Psychiatr Serv. 2009;60:880-3.

9. Le Berre A-P, Pitel A-L, Chanraud S, Beaunieux H, Eustache F, Martinot J-L, et al. Sensitive biomarkers of alcoholism's effect on brain macrostructure: similarities and differences between France and the United States. Front Hum Neurosci. 2015;9:354.

10. Segal JB, Bouffard MA, Schlaug G. Characteristic neuroimaging abnormalities of Korsakoff Syndrome. JAMA Neurol. 2016;73:1248-9.

11. Harper C, Dixon G, Sheedy D, Garrick T. Neuropathological alterations in alcoholic brains. Studies arising from the New South Wales Tissue Resource Centre. Prog Neuropsychopharmacol Biol Psychiatry. 2003;27:951-61.

12. Putzke J, De Beun R, Schreiber R, De Vry J, Tölle TR, Zieglgänsberger W, et al. Long-term alcohol self-administration and alcohol withdrawal differentially modulate microtubule-associated protein 2 (MAP2) gene expression in the rat brain. Brain Res Mol Brain Res. 1998;62:196-205.

13. Tarnowska-Dziduszko E, Bertrand E, Szpak GM. Morphological changes in the corpus callosum in chronic alcoholism. Folia Neuropathol. 1995;33:25-9.

14. Pfefferbaum A, Lim KO, Zipursky RB, Mathalon DH, Rosenbloom MJ, Lane $B$, et al. Brain gray and white matter volume loss accelerates with aging in chronic alcoholics: a quantitative MRI study. Alcohol Clin Exp Res. 1992; 16:1078-89.

15. Pfefferbaum A, Rosenbloom M, Rohlfing T, Sullivan EV. Degradation of association and projection white matter systems in alcoholism detected with quantitative fiber tracking. Biol Psychiatry. 2009;65:680-90.

16. Lewohl JM, Wang L, Miles MF, Zhang L, Dodd PR, Harris RA. Gene expressions in human alcoholism: microarray analysis of frontal cortex. Alcohol Clin Exp Res. 2000;24:1873-82.

17. Mamdani M, Williamson V, McMichael GO, Blevins T, Aliev F, Adkins A, et al. Integrating mRNA and miRNA weighted gene co-expression networks with eQTLs in the nucleus accumbens of subjects with alcohol dependence. PLoS One. 2015;10:e0137671 https://doi.org/10.1371/journal.pone.0137671
18. Osterndorff-Kahanek EA, Becker HC, Lopez MF, Farris SP, Tiwari GR, Nunez YO, et al. Chronic ethanol exposure produces time- and brain region-dependent changes in gene coexpression networks. PLoS One. 2015;10:e0121522 https://doi. org/10.1371/journal.pone.0121522

19. Harris GJ, Jaffin SK, Hodge SM, Kennedy D, Caviness VS, Marinkovic K, et al. Frontal white matter and cingulum diffusion tensor imaging deficits in alcoholism. Alcohol Clin Exp Res. 2008;32:1001-13.

20. Makris N, Oscar-Berman M, Jaffin SK, Hodge SM, Kennedy DN, Caviness VS, et al. Decreased volume of the brain reward system in alcoholism. Biol Psychiatry. 2008;64:192-202.

21. Ruiz SM, Oscar-Berman M, Sawyer KS, Valmas MM, Urban T, Harris GJ. Drinking history associations with regional white matter volumes in alcoholic men and women. Alcohol Clin Exp Res. 2013;37:110-22

22. Sawyer KS, Oscar-Berman M, Barthelemy OJ, Papadimitriou GM, Harris GJ, Makris N. Gender dimorphism of brain reward system volumes in alcoholism. Psychiatry Res. 2017;263:15-25.

23. Hamilton M. A rating scale for depression. J Neurol Neurosurg Psychiatry. 1960;23:56-62.

24. Abel EL, Kruger ML, Friedl J. How do physicians define "light," "moderate," and "heavy" drinking? Alcohol Clin Exp Res. 1998;22:979-84.

25. APA. Diagnostic and statistical manual of mental disorders DSM-IV-TR Washington: American Psychiatric Association; 2000.

26. Cahalan D, Cisin IH, Crossley HM. American drinking practices: a national study of drinking behavior and attitudes. New Haven: Publications Division, Rutgers Center of Alcohol Studies; distributed by College and University Press; 1969.

27. Robins LN, Cottler LB, Bucholz KK, Compton WM, North CS, Rourke KM. Diagnostic Interview Schedule for the DSM-IV (DIS-IV). St. Louis: Washington University School of Medicine; 2000.

28. Wechsler D. :. WAIS-III, Wechsler Adult Intelligence Scale, Third Edition: WMS-III, Wechsler Memory Scale, Third Edition: Technical Manual. San Antonio: Psychological Corporation; 1997. p. 426.

29. Smith SM, Jenkinson M, Woolrich MW, Beckmann CF, Behrens TEJ, Johansen-Berg $\mathrm{H}$, et al. Advances in functional and structural MR image analysis and implementation as FSL. Neuroimage. 2004;23(Suppl 1):S208-19.

30. Smith SM, Nichols TE. Threshold-free cluster enhancement: addressing problems of smoothing, threshold dependence and localisation in cluster inference. Neuroimage. 2009;44:83-98.

31. Zimmerman M, Martinez JH, Young D, Chelminski I, Dalrymple K. Severity classification on the Hamilton Depression Rating Scale. J Affect Disord. 2013;150:384-8.

32. Makris N, Meyer JW, Bates JF, Yeterian EH, Kennedy DN, Caviness VS. MRI-Based topographic parcellation of human cerebral white matter and nuclei II. Rationale and applications with systematics of cerebral connectivity. Neuroimage. 1999;9:18-45.

33. Makris N, Pandya DN. The extreme capsule in humans and rethinking of the language circuitry. Brain Struct Funct. 2009;213:343-58.

34. Makris N, Kennedy DN, Mclnerney S, Sorensen AG, Wang R, Caviness VS, et al. Segmentation of subcomponents within the superior longitudinal fascicle in humans: a quantitative, in vivo, DT-MRI study. Cereb Cortex. 2005;15:854-69.

35. Pfefferbaum A, Rosenbloom MJ, Adalsteinsson E, Sullivan EV. Diffusion tensor imaging with quantitative fibre tracking in HIV infection and alcoholism comorbidity: synergistic white matter damage. Brain. 2007;130:48-64.

36. Pfefferbaum A, Sullivan EV. Microstructural but not macrostructural disruption of white matter in women with chronic alcoholism. Neuroimage. 2002;15:708-18.

37. Pfefferbaum A, Sullivan EV. Disruption of brain white matter microstructure by excessive intracellular and extracellular fluid in alcoholism: evidence from diffusion tensor imaging. Neuropsychopharmacology. 2005;30:423-32.

38. Luo J, Chen G, Wei L, Qian H, Lai X, Wang D, et al. Severe diffuse axon injury in chronic alcoholic rat medulla oblongata following a concussion blow. Alcohol Alcohol. 2014;49:231-7.

39. Luo J, Shen Z, Chen G, Wang D, Yu X. Pontine changes in metabolites and axonal fibres of rats following four-week alcohol exposure: In vivo diffusion tensor imaging and 1h-magnetic resonance spectroscopy study at 7.0 T. Alcohol Alcohol. 2017;52:145-50.

40. Moffett JR, Ross B, Arun P, Madhavarao CN, Namboodiri AMA. N-Acetylaspartate in the CNS: from neurodiagnostics to neurobiology. Prog Neurobiol. 2007;81:89-131.

41. Squeglia LM, Sorg SF, Schweinsburg AD, Wetherill RR, Pulido C, Tapert SF. Binge drinking differentially affects adolescent male and female brain morphometry. Psychopharmacology. 2012;220:529-39.

42. Nicolai J, Moshagen M, Demmel R. Patterns of alcohol expectancies and alcohol use across age and gender. Drug Alcohol Depend. 2012;126:347-53.

43. Mosher Ruiz S, Oscar-Berman M, Kemppainen MI, Valmas MM, Sawyer KS. Associations between personality and drinking motives among abstinent adult alcoholic men and women. Alcohol Alcohol. 2017;52:496-505. 
44. Westlye LT, Bjørnebekk A, Grydeland H, Fjell AM, Walhovd KB. Linking an anxiety-related personality trait to brain white matter microstructure: diffusion tensor imaging and harm avoidance. Arch Gen Psychiatry. 2011;68:369-77.

45. White T, Nelson M, Lim KO. Diffusion tensor imaging in psychiatric disorders. Top Magn Reson Imaging. 2008;19:97-109.

46. Rilling JK, Glasser MF, Preuss TM, Ma X, Zhao T, Hu X, et al. The evolution of the arcuate fasciculus revealed with comparative DTI. Nat Neurosci. 2008;11:426-8.

47. Dick AS, Tremblay P. Beyond the arcuate fasciculus: consensus and controversy in the connectional anatomy of language. Brain. 2012;135:3529-50.

48. Pessoa L, Gutierrez E, Bandettini P, Ungerleider L. Neural correlates of visual working memory: fMRI amplitude predicts task performance. Neuron. 2002;35:975-87.

49. Sullivan EV. Contributions to understanding the neuropsychology of alcoholism: An INS legacy. J Intern Neuropsychol Soc. 2017;23:843-59.

50. Monnig MA, Yeo RA, Tonigan JS, McCrady BS, Thoma RJ, Sabbineni A, et al. Associations of white matter microstructure with clinical and demographic characteristics in heavy drinkers. PLoS One. 2015;10:e0142042.

51. Salat DH, Tuch DS, Greve DN, Kouwe AJWvander, Hevelone ND, Zaleta AK, et al. Age-related alterations in white matter microstructure measured by diffusion tensor imaging. Neurobiol Aging. 2005;26:1215-27.

52. Yang AC, Tsai S-J, Liu M-E, Huang C-C, Lin C-P. The association of aging with white matter integrity and functional connectivity hubs. Front Aging Neurosci. 2016;8:143.

53. Luhar RB, Sawyer KS, Gravitz Z, Ruiz SM, Oscar-Berman M. Brain volumes and neuropsychological performance are related to current smoking and alcoholism history. Neuropsychiatr Dis Treat. 2013;9:1767-84.

54. Durazzo TC, Pennington DL, Schmidt TP, Mon A, Abé C, Meyerhoff DJ. Neurocognition in 1-month-abstinent treatment-seeking alcohol-dependent individuals: interactive effects of age and chronic cigarette smoking. Alcohol Clin Exp Res. 2013;37:1794-803.

55. Meyerhoff DJ, Blumenfeld R, Truran D, Lindgren J, Flenniken D, Cardenas V, et al. Effects of heavy drinking, binge drinking, and family history of alcoholism on regional brain metabolites. Alcohol Clin Exp Res. 2004;28:650-61.

56. Gazdzinski S, Durazzo TC, Weiner MW, Meyerhoff DJ. Are treated alcoholics representative of the entire population with Alcohol Use Disorders? - A magnetic resonance study of brain injury. Alcohol. 2008;42:67-76.
57. Harris GJ, Oscar-Berman M, Gansler DA, Streeter C, Lewis RF, Ahmed I, et al. Hypoperfusion of cerebellum and aging effects on cerebral cortex blood flow in abstinent alcoholics: A SPECT study. Alcohol Clin Exp Res. 1999; 23:1219-27.

58. Oscar-Berman M, Clancy JP, Weber DA. Discrepancies between IQ and memory scores in alcoholism and aging. Clin Neuropsychol. 1993;7:281-96.

59. Papademetris X, Jackowski MP, Rajeevan N, DiStasio M, Okuda H, Constable RT, et al. Biolmage suite: an integrated medical image analysis suite: an update. Insight J. 2006;2006:209.

60. Compton WM, Conway KP, Stinson FS, Colliver JD, Grant BF. Prevalence, correlates, and comorbidity of DSM-IV antisocial personality syndromes and alcohol and specific drug use disorders in the United States: results from the national epidemiologic survey on alcohol and related conditions. J Clin Psychiatry. 2005;66:677-85.

61. Jagannathan NR, Desai NG, Raghunathan P. Brain metabolite changes in alcoholism: an in vivo proton magnetic resonance spectroscopy (MRS) study. Magn Reson Imaging. 1996;14:553-7.

62. Keyes KM, Hatzenbuehler ML, Hasin DS. Stressful life experiences, alcohol consumption, and alcohol use disorders: the epidemiologic evidence for four main types of stressors. Psychopharmacology. 2011;218:1-17.

63. Khan S, Okuda M, Hasin DS, Secades-Villa R, Keyes K, Lin K-H, et al. Gender differences in lifetime alcohol dependence: results from the national epidemiologic survey on alcohol and related conditions. Alcohol Clin Exp Res. 2013;37:1696-705.

64. Kuntsche E, Knibbe R, Gmel G, Engels R. Who drinks and why? A review of sociodemographic, personality, and contextual issues behind the drinking motives in young people. Addict Behav. 2006;31:1844-57.

65. Nolen-Hoeksema S, Hilt L. Possible contributors to the gender differences in alcohol use and problems. J Gen Psychol. 2006;133:357-74.

66. Oscar-Berman M, Valmas MM, Sawyer KS, Kirkley SM, Gansler DA, Merritt D, et al. Frontal brain dysfunction in alcoholism with and without antisocial personality disorder. Neuropsychiatr Dis Treat. 2009;5:309-26.

67. Parks MH, Dawant BM, Riddle WR, Hartmann SL, Dietrich MS, Nickel MK, et al. Longitudinal brain metabolic characterization of chronic alcoholics with proton magnetic resonance spectroscopy. Alcohol Clin Exp Res. 2002;26: 1368-80. 